\title{
Bernhard Bergmans, Grundlagen der Rechtsdidaktik an Hochschulen, Band 1: Rechtsdidaktik als Wissenschaft und Praxis, Berlin 2014, 141 S., 29,00 Euro
}

\section{Nora Rzadkowski"}

Während der letzten Jahre ist ein bunter Strauß an rechtsdidaktischen Themen und Forschungsansätzen auf Tagungen, in rechtsdidaktischen Zentren und neuerdings in der ZDRW präsentiert worden. Eine Systematisierung verspricht Bergmans mit seinen „Grundlagen der Rechtsdidaktik an Hochschulen“. Das ambitionierte Projekt soll in vier Bänden verwirklicht werden. Der erste Band liegt nunmehr vor und ist Gegenstand der Rezension. ${ }^{1}$

Im ersten Teil des Projekts will Bergmans die Rechtsdidaktik als Wissenschaft etablieren. ${ }^{2}$ Der Band beginnt mit einer Bestandsaufnahme (I.): Der nicht zufriedenstellende Status quo der Rechtsdidaktik wird beleuchtet, Gründe erörtert, positive Entwicklungen aufgezeigt und ein vergleichender Blick ins Ausland gewagt. Es folgt eine Grundkonzeption der Rechtsdidaktik (II.), in der Bergmans den Begriff der Rechtsdidaktik erläutert, Gegenstand und Aufgaben der Rechtsdidaktik systematisiert und schließlich Abgrenzungen zu Gebieten wie der Rechtspädagogik, der Rechtslehre oder der juristischen Methodik vornimmt. Anschließend geht Bergmans nochmals auf die Notwendigkeit und den Nutzen der Rechtsdidaktik ein (III.). Die Rechtsdidaktik hält er beispielsweise für unerlässlich, um das Auseinanderklaffen zwischen juristischer Ausbildung und gesellschaftlicher Entwicklung zu überwinden, die Inanspruchnahme öffentlicher Ressourcen zu rechtfertigen und mit der Heterogenität der Studierenden besser umgehen zu können. Kapitel IV widmet sich dann dem Hauptanliegen des Bandes, der Wissenschaftlichkeit der Rechtsdidaktik, während das letzte Kapitel sich mit der Implementierung der Rechtsdidaktik befasst (V.). Dabei werden die Rollen der Lernenden und Lehrenden sowie die Rahmenbedingungen näher beleuchtet.

Die Leistung des Bandes liegt sicherlich im Versuch einer Bestimmung und Systematisierung der Rechtsdidaktik an Hochschulen. Dabei ist es auch interessant, dass der Autor als Fachhochschullehrer die Perspektive erweitert: Er nimmt nicht nur das klassische Universitätsstudium in den Blick, sondern ein differenziertes Ausbildungsund Studienangebot. Bei einer so großen Aufgabe dürfen die Erwartungen sicher nicht zu hoch gesteckt werden - das Ergebnis bleibt trotzdem unzureichend. Ich verstehe es als Aufgabe einer fachspezifischen Hochschuldidaktik, einerseits Verbindungen zur allgemeinen Didaktik bzw. Hochschuldidaktik herzustellen, zum anderen die Spezifika des Faches herauszuarbeiten, die eine Anpassung oder Weiterentwicklung erforderlich machen. Bergmans arbeitet die Erkenntnisse anderer Diszipli-

* Ass. iur. Nora Rzadkowski, MoHE ist Doktorandin an der Albrecht Mendelssohn Bartholdy Graduate School of Law in Hamburg.

1 Die weiteren Bände sollen folgende Inhalte haben: Band 2: Ziele, Inhalte und Strukturen, Band 3: LehrLern-Modelle, Methoden und Instrumente, Band 4: Tests, Prüfungen und Evaluationen.

2 S. 7. 
nen nicht auf. Oder zumindest werden diese nicht zitiert. ${ }^{3}$ Ausländische rechtsdidaktische Literatur wird kaum berücksichtigt, obwohl Bergmans bei allen Zweifeln an der Übertragbarkeit der Konzepte den „Blick über den Zaun“ selbst befürwortet. ${ }^{4}$ Gleichwohl versichert er: „Der Leser kann jedoch davon ausgehen, dass nicht zitierte Literatur keinen inhaltlichen Mehrwert bietet. " ${ }^{5}$ Eine steile These. Mit Kritik an Kollegen und Kolleginnen spart Bergmans nicht. ${ }^{6}$ Mag sie im Einzelnen auch berechtigt sein: Lust zum Lesen und zur Weiterentwicklung der eigenen Lehre macht sie nicht gerade. Aber das ist vielleicht auch nicht Zweck des Bandes, der sich weniger an die Lehrenden als an den Kreis der Rechtsdidaktikerinnen und -didaktiker wendet.

Diese Zielsetzung aufnehmend werde ich mich im Folgenden mit zwei Vorschlägen auseinandersetzen, die ich als zentral für Bergmans Band und die weitere Entwicklung der Rechtsdidaktik halte. Dabei handelt es sich zum einen um die Benennung des Forschungsgebiets und zum anderen um den Status der Wissenschaftlichkeit, den Bergmans für die Rechtsdidaktik einfordert.

Didaktik der Rechtswissenschaft, Rechtswissenschaftsdidaktik, rechtswissenschaftliche Fachdidaktik oder Rechtsdidaktik - welcher Name eignet sich am besten für das (wieder) entstehende Forschungsgebiet? Wie schon aus dem Titel des Bandes hervorgeht, entscheidet sich Bergmans für den Begriff der Rechtsdidaktik. Bergmans ist sicherlich zuzustimmen, dass die „sprachökonomische Griffigkeit" ${ }^{7}$ größer ist als bei den alternativen Vorschlägen. Aber es geht ja nicht nur um Begrifflichkeiten, sondern darum, das Forschungsfeld abzustecken. Was hat also andere Autorinnen und Autoren bewogen, Wortungetümen wie der „Didaktik der Rechtswissenschaft“ oder der „rechtswissenschaftlichen Fachdidaktik“ den Vortritt zu gewähren? ${ }^{8}$ Dafür werden zwei Gründe angeführt: Zum einen geht es um Klarheit. Während Rechtsdidaktik mit dem Lernort Schule assoziiert wird, sollen die Alternativen deutlich machen, dass das Lehren und Lernen an Hochschulen gemeint ist. Zum anderen wird eine inhaltliche Forderung deutlich: nicht nur Recht soll Gegenstand der Lehre sein, sondern auch die Rechtswissenschaft.

3 Viele Fußnoten enthalten zudem interne Verweisungen, wie „S. hierzu Band 2“ - was derzeit nicht überprüfbar ist und auch bei Erscheinen der weiteren Bände zu einiger Sucharbeit führen dürfte.

4 S. 54 .

5 S. 8 Fn. 2.

6 Eine kleine Auswahl: „[...] die Mehrzahl der Hochschuldozenten ist überzeugt, sie seien für die Lehre gut qualifiziert. Diese Selbstüberschätzung hängt vermutlich mit den unterschiedlichen Anspruchsniveaus und Bewertungsmaßstäben für die Qualität von Forschung und Lehre zusammen“ (S. 108); „Professoren sind didaktische Dilettanten, auch wenn die Lehrfähigkeit mit der Erfahrung zunimmt“" (S. 20); „Es beteiligen sich auch zahlreiche Lehrende an den Diskussionen, aber diese [...] erkennen mangels Qualifikation nicht die Tragweite der Thematiken und diskutieren unter ihrem intellektuellen Niveau (ohne es aber offenbar zu merken) [...]“ (S. 79).

7 S. 33.

8 Pilniok/Brockmann/Dietrich, Juristische Lehre neu denken: Plädoyer für eine rechtswissenschaftliche Fachdidaktik, in: Brockmann/Dietrich et al. (Hrsg.), Exzellente Lehre im juristischen Studium: Auf dem Weg zu einer rechtswissenschaftlichen Fachdidaktik, Baden-Baden 2011, S. 9 (16); Pilniok, Rechtswissenschaftliche Fachdidaktik als Reflexion der Rechtswissenschaft, in: Griebel/Gröblinghoff (Hrsg.), Von der juristischen Lehre, Baden-Baden 2012, S. 17 (22). 
Dagegen wendet Bergmans ein, dass sich die Situierung sowieso schon aus dem jeweiligen Kontext ergebe. Wenn an Hochschulen von Rechtsdidaktik gesprochen werde, sei ja klar, dass die Rechtsdidaktik an Hochschulen gemeint sei. Rechtsdidaktik ist für ihn darüber hinaus ein Oberbegriff, unter den auch die rechtswissenschaftliche Fachdidaktik fällt. Eine Differenzierung zwischen der Vermittlung von Recht und Rechtswissenschaft hält er für weitgehend unnötig: Die Grundfragen seien, egal in welcher Lehrinstitution, in der Regel dieselben. ${ }^{9}$ Die angebliche Wissenschaftlichkeit des Studiums wird als Label verstanden, das kaum praktische Relevanz hat: „Die Wissenschaftlichkeit als gedanklicher Überbau des Rechts und seiner Methoden ist nur eine Ergänzung, deren ideeller Wert zwar unstrittig ist, deren tatsächliche Bedeutung in der Lehre jedoch überschaubar bleibt. "10 An den Fachhochschulen und beim Studium des Rechts als Nebenfach spiele die Wissenschaftlichkeit sowieso eine sehr untergeordnete Rolle.

Dazu drei kritische Anmerkungen. Ich stimme Bergmans zu, dass Rechtsdidaktik nicht notwendig mit dem Lernort Schule assoziiert werden muss. „Rechtsdidaktik“ kann als Lehren und Lernen von Recht unabhängig von spezifischen Situierungen und Organisationsformen verstanden werden. Dann eröffnet sich jedoch ein weites Feld der Rechtsdidaktik: Kinder lernen, wie sie Verträge abschließen, Verbraucher informieren sich über ihre Rechte in Internetforen, die Medien erklären die aktuelle Rechtsprechung oder in der anwaltlichen Beratung muss die Rechtslage in Hinblick auf den konkreten Fall erörtert werden. Hier ließen sich eine Vielzahl interessanter Fragen in Hinblick auf die Vermittlung von Recht entwickeln. Diese weite Perspektive nimmt Bergmans aber nicht ein. Er entfaltet den Begriff und die Reichweite der Rechtsdidaktik nicht, sondern benutzt den Begriff als kleinsten gemeinsamen Nenner, unter dem sich Universitäts- und Fachhochschulstudium zusammenfassen lassen. Zweitens teile ich Bergmans Auffassung nicht, dass sich überall die gleichen Grundfragen stellen. Bergmans selbst weist immer wieder auf die ausdifferenzierte Studien- und Ausbildungslandschaft hin, die von der Rechtsdidaktik bisher wenig berücksichtigt worden ist. In unterschiedlichen Kontexten werden unterschiedliche Zielsetzungen verfolgt, die sich auch in der Rechtsdidaktik widerspiegeln müssen. Es macht einen Unterschied, ob es darum geht, Studierenden der Sozialarbeit Rechtskenntnisse für ihr berufliches Handeln zu vermitteln oder Doktoranden und Doktorandinnen dabei zu helfen, das Recht kritisch zu beleuchten oder Vorschläge zu dessen Weiterentwicklung zu erarbeiten. Drittens: Die Beobachtung, dass die Wissenschaftlichkeit in der Realität des Hochschulstudiums kaum eine Rolle spielt, ist wahrscheinlich realistisch. ${ }^{11}$ Angesichts dieser Situation für eine Rechtsdidaktik zu votieren, den Wissenschaftsbezug also aus dem Namen zu streichen, könnte als desillusionierendes, vielleicht auch provokatives Statement verstanden werden. Dafür fehlt Bergmans aber der kritische Impetus, den eine (Hochschul-)Didaktik haben

9 S. 33: „viele Grundfragen [sind] (rechts-)fachbezogen und unabhängig von der Lehrinstitution bzw. Person der Lernenden “.

10 S. 34 .

11 Letztlich ist die Einschätzung abhängig vom jeweiligen Verständnis von Rechtswissenschaft. 
sollte und die im Beharren auf einer rechtswissenschaftlichen Fachdidaktik meines Erachtens zum Ausdruck kommt. ${ }^{12}$

Während Bergmans zwischen Recht und Rechtswissenschaft keinen wirklichen Unterschied sieht, ist es sein erklärtes Ziel, die Rechtsdidaktik als Wissenschaft zu etablieren. Bergmans hält dies für notwendig, weil das fehlende Niveau der derzeitigen, häufig auf anekdotischer Evidenz und Einzelerfahrung basierenden Rechtsdidaktik die Gefahr berge „im Dilettantismus zu verharren“. ${ }^{13}$ Wissenschaftlichkeit versteht er als Zeichen der Professionalität, die der Rechtsdidaktik auch zu einer höheren Akzeptanz verhelfen könne. ${ }^{14} \mathrm{Er}$ sieht in ihr eine „an den Maßstäben der Rationalität, Objektivität, argumentativen Begründung und des methodischen Vorgehens ausgerichtete intellektuelle Beschäftigung " ${ }^{15}$ die sich an der Realität orientiert und auf praxisrelevante Erkenntnisse zielt. Auf sachfremde, insbesondere politische Wertungen sei zu verzichten. ${ }^{16}$ Insgesamt sei die Rechtsdidaktik nicht in der Rechtswissenschaft zu verorten, sondern als „nicht-juristische Wissenschaft, die sich (auch) dem Phänomen des Rechts und der Rechtswissenschaft widmet" ${ }^{17}$ zu verstehen; „Untersuchungsziele, -methodik und -sprache der Rechtsdidaktik sind gerade nicht die der Rechtswissenschaft, sondern die der Didaktik " ${ }^{18}$ betont Bergmans.

Es erstaunt, dass Bergmans die Rechtsdidaktik so eindeutig disziplinär in der Didaktik verankert, es aber versäumt, die didaktische Literatur auszuwerten, die sich mit dem Status der Didaktik als Wissenschaft, ihren Paradigmen und Forschungsmethoden befasst. Die Lektüre hätte folgende Punkte erhellen können:

Der Status der Didaktik als Wissenschaft ist nicht weniger problematisch als der der Dogmatik. Die Didaktik oszilliert zwischen Theorie und Praxis. Ihre Ergebnisse können „Zwischenformen“ annehmen, wie etwa didaktische Modelle oder Konzepte, die theoretische Entwürfe aufnehmen, sich aber um eine handlungsanleitende Aufbereitung bemühen. ${ }^{19}$ Mit anderen Worten: Die Frontstellung von persönlichen Er-

12 Die Metapher der Wissenschaftlichkeit als „gedanklichem Überbau des Rechts“ ist zudem Ausdruck der Verquickung von Recht und Rechtswissenschaft, die in der deutschen Rechtswissenschaft zwar üblich ist, aber zunehmend in die Kritik geraten ist. Denn ihr „ideeller Wert“ ist keinesfalls unstrittig. Sie verschleiert die Potentiale rechtswissenschaftlicher Forschung, die nicht unter den Entscheidungszwängen der Praxis steht. Vgl. dazu etwa Lepsius, Kritik der Dogmatik, in Kirchhof/Magen et al. (Hrsg.), Was weiß Dogmatik?, Tübingen 2012, S. 39 (60 f.); Peczenik, Scientia Juris, Dordrecht u.a. 2007, 156 ff. Kritische Autorinnen und Autoren sehen in der Betonung der wissenschaftlich-rationalen Überformung des Rechts außerdem die Gefahr, die Dominanz westlichen Rechtsdenkens zu perpetuieren. Vgl. Darian-Smith, Laws and Societies in Global Contexts: Contemporary Approaches, Cambridge 2013, S. 48 ff., 97 ff.; Santos, Beyond abyssal thinking. From global lines to ecologies of knowledges, Review (Fernand Braudel Center) 2007, S. 45 ff.

13 S. 75.

14 S. 75.

15 S. 80.

16 Es folgen längere Ausführungen zu Werten und Leitbildern, die sich in der juristischen Ausbildung wie in der Rechtsdidaktik niederschlügen und die wohl für die „Rationalität und Objektivität“ sensibilisieren sollen.

17 S. 93.

18 S. 93.

19 Kron, Wissenschaftstheorie für Pädagogen, München/Basel 1999, S. 77 f. 
fahrungen und Wissenschaftlichkeit ist kontraproduktiv. Oft sind Erfahrungen Ausgangspunkt didaktischer Forschung. Und die Ergebnisse didaktischer Forschung wirken sich idealerweise wiederum auf das Handeln der Lehrenden und Lernenden aus. Neuere hochschuldidaktische Modelle nehmen diese Idee auf. So zum Beispiel das „Scholarship of Teaching and Learning“, das die Reflexion des eigenen Lehrhandelns mit wissenschaftlichen Mitteln anregt. ${ }^{20}$ Die Forschungsparadigmen der Erziehungswissenschaft werden heute als unterschiedliche Ausrichtungen verstanden; „Richtigkeit“ oder „Wahrheit“ wissenschaftlicher Erkenntnisse lassen sich nur relativ zum jeweils gewählten Paradigma und Erkenntnisinteresse bestimmen. ${ }^{21}$

Ob die Rechtsdidaktik Teil der Rechtswissenschaft oder der Didaktik ist - auch dazu hätte man in der (hochschul-)didaktischen Literatur einiges finden können. ${ }^{22} \mathrm{Berg}$ mans einseitige Zuordnung der Rechtsdidaktik zur Didaktik blendet die eigentliche Herausforderung aus: das Herstellen von Bezügen und Kooperationsmöglichkeiten zwischen einer Vielzahl von (Sub-)Disziplinen. Denn die Didaktik ist selbst ein „buntscheckiges Gemisch“, ${ }^{23}$ das über unterschiedliche disziplinäre Anbindungen, Forschungsparadigma und -methoden verfügt. Die Verunsicherung des Dazwischen, des Weder/Noch ist in meinen Augen stimulierender als eindeutige Zuordnungen und führt zu einem Verständnis der Rechtsdidaktik als transdisziplinärem Forschungsfeld.

Alles in allem kann man darüber streiten, ob der Band die Grundlagen der Rechtsdidaktik an Hochschulen ausreichend beschreibt. Zumindest stößt er Diskussionen über sie an und das ist durchaus erfreulich.

20 Huber, Forschen über (eigenes) Lehren und studentisches Lernen - Scholarship of Teaching and Learning (SoTL). Ein Thema auch hierzulande?, in: Das Hochschulwesen 2011, S. $118 \mathrm{ff}$.

21 Kron, Wissenschaftstheorie für Pädagogen, München/Basel 1999, S. 263 ff.

22 Für die Hochschuldidaktik stellt Wildt, Ein Blick zurück - Fachübergreifende und/oder fachbezogene Hochschuldidaktik: (K)eine Alternative?, in: Jahnke/Wildt (Hrsg.), Fachbezogene und fachübergreifende Hochschuldidaktik, Bielefeld 2011, S. 19 (22) bspw. fest: „Die Hochschuldidaktik setzt an der Differenz zwischen fachlichen und didaktischen Diskursen an. "Vgl. auch Plöger, Allgemeine Didaktik, Fachdidaktik, Fachwissenschaft, in: Hellekamps/Plöger et al. (Hrsg.), Handbuch der Erziehungswissenschaft, Bd. 3, Paderborn 2011, S. 427 ff. und die dort folgenden Beiträge, in denen das Wechselspiel zwischen didaktischen und fachwissenschaftlichen Anteilen in einzelnen Fachdidaktiken exemplarisch nachgezeichnet wird.

23 Der Begriff wird von Gudjons, Pädagogisches Grundwissen, Bad Heilbrunn 2012, S. 19 mit Blick auf die Erziehungswissenschaft verwendet. 